\title{
Military and Medical Expertise in Patients with Central Retinal Degeneration
}

\author{
Ignatiev Sergei ${ }^{1}$, Alekseev Igor ${ }^{2,3}$, Nam Yuliya ${ }^{3}$ \\ ${ }^{1}$ Department of Diagnostics, City Clinical Hospital Named by S. P. Botkin of the Department of Health, Moscow, Russian Federation \\ ${ }^{2} 4^{\text {th }}$ Surgical Department of Branch № 1 of the City Clinical Hospital named by S. P. Botkin of the Department of Health, Moscow, Russian \\ Federation \\ ${ }^{3}$ Department of Ophthalmology, Federal Budgetary State Educational Institution of the Additional Professional Education Russian Medical \\ Academy of Continuous Post-Graduate Education, Moscow, Russian Federation
}

Email address:

yuliyanam@rocketmail.com (N. Yuliya)

\section{To cite this article:}

Ignatiev Sergei, Alekseev Igor, Nam Yuliya. Military and Medical Expertise in Patients with Central Retinal Degeneration. International Journal of Clinical and Experimental Medical Sciences. Vol. 4, No. 4, 2018, pp. 68-72. doi: 10.11648/j.ijcems.20180404.13

Received: September 17, 2018; Accepted: October 24, 2018; Published: November 14, 2018

\begin{abstract}
Last years pathological processes of the retina became a worldwide problem among people of different ages and this group includes a various number of central retinal degenerations. There is a vivid tendency for these types of diseases to become younger last years. Such changes are the most common reasons of vision loss, especially age-related macular degeneration and its exudative form. Recent studies show that this form of AMD is linked not only with aging, weakness of the vessels and free radical oxidation, but also with non-specific immune stimulation and the potential role as a trigger of cytomegalovirus infection. No wonder, that chronic infection of endothelial cells leads to attracting to inflammation region of macrophages, their adhesion and synthesis of anti-inflammation cytokines and after all if stimulates formation or activation of CNV. Some pathogenetic peculiarities, methods of treatment and medical expertise are described in this article. Still, they are in the first rate in studies of ophthalmologists all over the world. Social meaning of this pathology is important due to the developing of disability in population and especially in military personnel. The article provides chronic, difficult curable or incurable diseases and outcomes of injuries and burns of the eyes. The matter is that in cases with a completed process or a progressing course with rare exacerbations (less than 2 times per year), the category of fitness for military service is determined depending on the eye functions strictly in accordance with the relevant articles of the disease schedule.
\end{abstract}

Keywords: Age-Related Macular Degeneration, Medical Expertise, Military Expetise

\section{Introduction}

Despite of the achievements of modern medicine pathological processes of the eye fundus including central retinal degeneration are the most common reason of worsening and loss of the vision. Among all the types of the central retinal degeneration age-related macular degeneration (AMD) is the most often and it is still the leading cause of irreversible vision loss and blindness among the population in developed countries at the age of 50 and older.

In the Russian Federation the morbidity of AMD is more than 15 cases per thousand. In the structure of the initial disability due to AMD the number of affected people able to work is $21 \%$ and $32 \%$ are pensioners. In connection with the expected increasing of life expectancy, continuing growth of atherosclerosis and concomitant pathology, the problem of AMD seems to be interesting. There is a vivid tendency for this disease to become younger last years. Social meaning of this pathology is important due to the fast central vision loss and developing of disability in people especially in military personnel $[6,8]$.

\section{The Connection Between AMD and Cytomegalovirus (CMV)}

Without any exaggeration the last 10 years can be called as "AMD era" because the question about its etiology and pathogenesis is actively discussed in scientific forums, in 
printed publications and in internet. Domestic and foreign ophthalmologists unanimously suppose AMD as multifactorial disease and aging, smoking, ethnical belonging, hereditary factors etc. play role for its development [2].

Among the first eight pathogenic representatives of Herpesviridae family which played the role in developing of chronic vessel pathology was CMV. Fabricant et al. (1973) first presented the theory about the connection of atherosclerosis with Herpes viruses. In research works they detected the ability of the virus to violate lipid exchange in animals' endothelial cells and cause deposition of cholesterol in them with the further formation of atherosclerotic plaques. These studies acted like a stimulus for definition of the role of CMV infection in developing of such socially significant diseases like ischemic heart disease, acute violation of coronary circulation, Alzheimer's diseases and AMD.

CMV is widespread among people. The part of seropositive persons in Russian adults is $73-98 \%$ [3]. After virus overcoming of entrance gates (any mucosal tissues), it enters the blood flow and then spread by macrophages and lymphocytes to organs and tissues [4]. CMV is able to set chronical latent infection, which has a paradox feature: high levels of defeating antibodies are connected with asymptomatic multiply-active virus that can be detected in different cell cultures (epithelial, endothelial cells, fibroblasts, neuroglial and muscle cells, leucocytes, macrophages). Due to low pathogenic activity of the virus and its ability to suppress cellular immunity (after long evolution and virus adaptation for human body), lifelong CMV persistence appears in "healthy" persons with a periodic reactivation of the infection which is usually subclinical [13, 14].

Special attention should be paid to reproduction of the virus in endothelial cells that are the layer of vessels in all organs and tissues of the human body. Because of that fact CMV is able to permeate in parenchymal cells of any organ. The role of $\mathrm{CMV}$ as an inflammation trigger with the following developing of vessel pathology (atherosclerosis, vasculitis) was confirmed by many domestic and foreign researchers. Some authors suggest a probable role of CMV in developing AMD and transition from dry to wet form by revealing high levels of $\mathrm{IgG}$ to $\mathrm{CMV}$ in blood serum in patients with age related changes of macular region [7].

Different authors in their research works with rodents made attempts to find the connection between chronic systemic CMV infection and developing of the CNV. Sarcs et. al (1999), Espinosa-Heidemann et al. (2003) in their experiments on animals revealed the role of macrophages infected with CMV in formation of neovascular membrane.

The basis of this process takes part in long persistence of CMV in macrophages with supporting latent infection. Initial CMV infection usually occurs in childhood and is accompanied by creating of constant latent infection of hematopoietic stem cells - bone marrow precursors (monocytes in our case). After maturation of monocytes infected with CMV gene expression occurs and cell becomes "aggressive". Highly effective infected monocytes migrate and after reaching choriocapillaries and pigment epithelial cells they produce inflammation mediators as an answer to laceration. Though, what makes cells of monocyte row migrate from the peripheral blood to choricapillaries is still unclear. Antimacrophage therapy (medications which reduces monocytes circulating in the blood) results in the decrease of size and activity of neovascular membrane [13, 14].

Alternative explanation of CMV role in CNV development is infection of endothelial cells of choriocapillaries. Research works revealed that periodic reactivation of latent $\mathrm{CMV}$ infection is able to lead for inflammation of circulating monocytes and neutrophils which act as carriers of infection virions to endothelial cells. Stable infection on endothelial cells leads to attracting to inflammation region of macrophages, their adhesion and synthesis of antiinflammation cytokines and also triggers the formation or activation of CNV [12].

The third explanation of CNV influence to exudative AMD formation is linked with non-specific immune stimulation. It is known that most of persistent virus infections are characterized with disturbance of cytokine net functioning. The basis of pathogenesis of long term location of pathogens in organism is because of marked imbalance of Thll and Thl2 ways of immune response and dysregulation of production, interaction of produced cytokines. Thus, the scenery of the development of AMD in every organism is determined by age related changes of vessel system and pigment epithelial cells, genetic predisposition, mode of life, nutrition and metabolism, immune response and many other factors, being, thereafter, a multifactorial process. We suppose that main pathogenesis chains in AMD development are shown in figure 1.

Along with other diseases, connected with degenerative age related processes (atherosclerosis, Alzheimer's disease, ischemic heart diseases) immune and inflammation response mechanisms are vividly seen in pathogenesis of AMD and get features of justified scientific theory, approved by facts of imbalance of immune system in the local and systemic levels. Not the last role in immune-mediated inflammation in AMD, probably, play infectious agents and special place in this case occupies chronic CMV infection. Thus, CMV infection is a trigger which forces to support the maintenance and progression of inflammation.

The treatment of people with AMD is still a serious problem of modern ophthalmology. Majority of authors accept the fact that conservative and surgical treatment in most patients with AMD is not very effective. Research works show the increasing of visual functions (approximately for 0,2-0,3 in Snellen's chart) and its stabilization after using traditional conservative treatment in $35 \%$ of patients (in other $45 \%$ of patients the treatment is non-effective). By the way $20 \%$ of patients show the progression of AMD with the loss of visual functions $[5,6]$. For this reason the finding of new means and methods of treatment is actual, especially affecting on different chains of pathogenesis of degenerative retinal diseases. This treatment should lead to the restoration 
of chorioretinal blood flow and support of viable nerve fibers and retinal fibers in its sclerotic lesion.

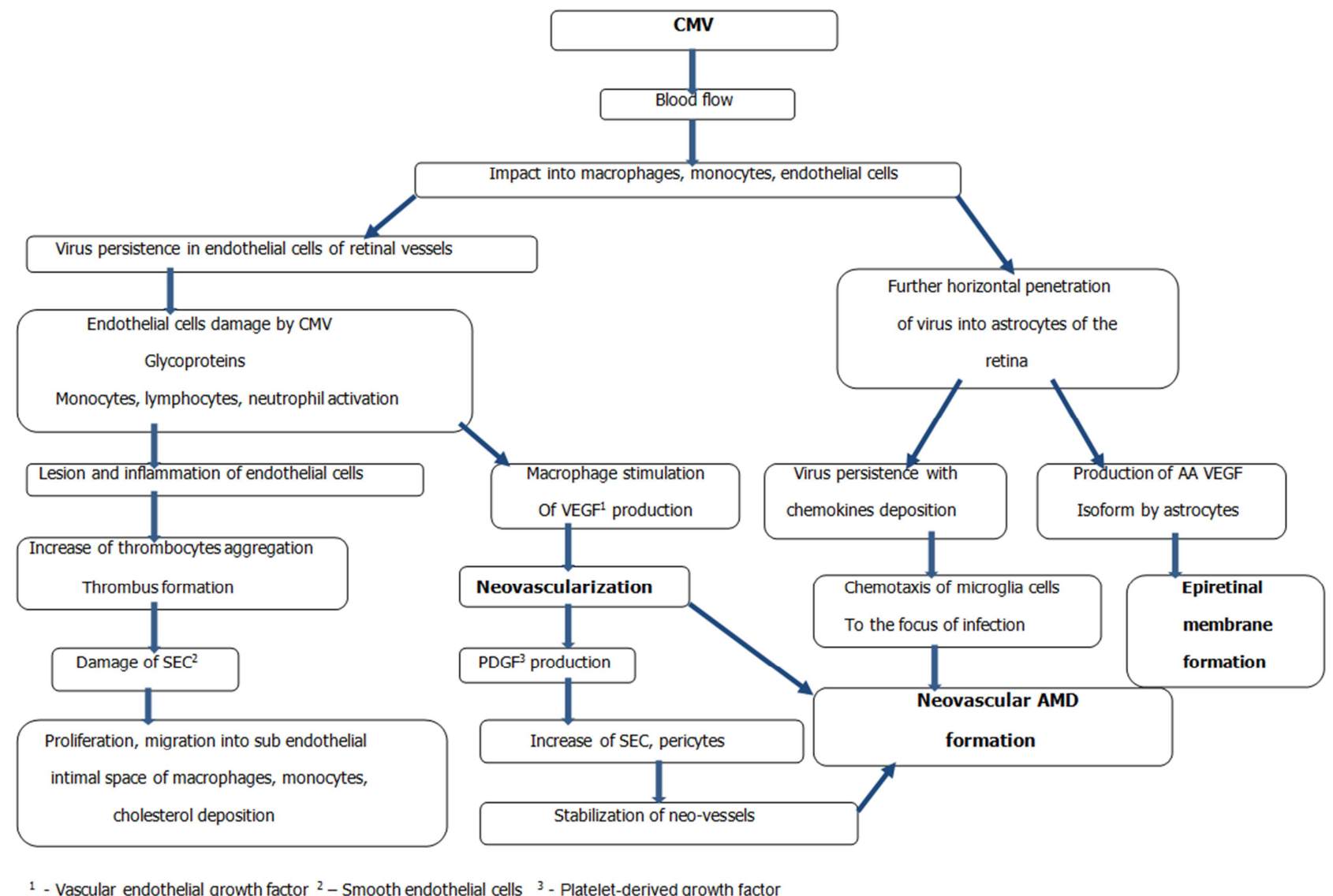

Figure 1. Pathogenesis of AMD development according to connection with CMV.

Studying the infectious theory of AMD development where the leading role plays inflammatory factor with following endothelial dysfunction (atherosclerosis, vasculitis) some NSAIDs show their effectiveness in the instilling shape.

Preference is given to eye drops with the ability to permeate to the posterior pole of eye. In case of endothelial dysfunction the main points of treatment are restoration of the balance, limitation of acting of some endothelial mediators and compensation of other deficiency.

Nitrates restore the deficit of nitrogen oxides (NO), inhibitors of the angiotensin converting enzyme block the synthesis of angiotensin II and prevent the destruction of kinins, calcium antagonists inhibit the activity of angiotensin II and endothelin in the smooth muscle of the vessels, enhancing the vasodilating effect of NO, endothelinconverting enzyme and inhibitors of endothelin receptors block the action of this peptide.

Endothelium is the main target of atherogenesis. Lipids are unable to penetrate through intima, but barrier function of endothelium changes because of risk factors - smoking, hypercholesterolemia, hyperinsulinemia, hypertension, aging, menopause and probably under the condition of systemic and local inflammation mediators, producing by endothelium. Among others, the most significant effect of drugs decreasing the level of lipids is their ability to improve endothelial function. In people with hypercholesterolemia simvastatin improves originally disturbed endothelial function.

\section{Methods of Treatment of AMD}

There is rather popular therapy of non-exudative AMD form by prescribing vitamins with antioxidant activity, lutein, zeaxanthin and zinc. Therapy is aimed to the prevention of new druses formation, lipofuscin deposition, pigment migration and focuses of atrophy.

Significant changes in AMD treatment are linked with appearance of peptide bioregulators acting in protein synthesis and regulation of cellular metabolism. The most well-known effective byopeptide is Retinalamin. It is a complex of low molecular polypeptides with molecular mass from 1000 to 10000 daltons, that is enough to penetrate through blood-ocular barrier. Its main action is in photoreceptors and retinal cells stimulation, acceleration of processes of restoration of light sensitivity improvement of the function of the viability of pigment epithelium and photoreceptors, stimulation of reparative processes [11].

The main direction of the treatment of exudative (wet) AMD form is an impact on choroidal neovascularization, prevention of new vessels formation and promotion of vessel wall permeation. A new period in treatment of neovascularization in AMD has come with appearance of 
VEGF inhibitors. They became the first choice methods. Main mechanism of their action is linking and inactivation of biologically active endothelial growth factor A (VEGF), inducing the growth of neovascular vessels.

There were determined clear indications for photodynamic therapy in treatment of exudative AMD form with usage of low-energy laser irradiation of the affected area of the retina after preliminary intravenous injection of verteporfirin.

As a result of photochemical decomposition of the drug, atomic oxygen is released, which damages endothelial cells, leading to occlusion of newly formed vessels [10].

Laser coagulation using in exudative and hemorrhagic stage leads to destruction of retinal tissues and scar formation. The main principle of laser intervention is not to damage fovea and this reduce the number of patients in which such treatment is possible to be used.

There are three known surgical methods of treatment and two of them may lead to serious complications if there is a doubtful possibility of a positive effect. Thus, deleting of well bordered neovascular membrane may cause choriocapillary atrophy. Retinal translocation may also cause choriocapillary atrophy and proliferative vitreoretinopathy. Transplantation of pigment epithelial cells is still at the stage of experimental studies.

\section{Medical Expertise}

Military and medical expertise in military personnel with AMD is carried out in case of the determined medical and expert outcome which means - after achieving of such health condition, when results of diagnostics and treatment allow to make a decision about category of fitness for military service and when the further treatment won't change it.

Medical expertise of military personnel with AMD is carried out on the base of requirements according to article №30 of diseases schedule (application to the Provision on military medical examination approved by the resolution of the Russian Federation Government on July 42013 №565). The article provides chronic, difficult to curable or incurable diseases and outcomes of injuries and burns of the eyes. In cases with a completed process or a progressing course with rare exacerbations (less than 2 times per year), the category of fitness for military service is determined depending on the functions of the eye in accordance with the relevant articles of the disease schedule. AMD is characterized with loss of central vision, that's why medical examination is conducted in accordance with the visual acuity requirements according to the article №35 of the diseases schedule. Visual acuity is taken into account with correction by any glasses, as well as contact lenses in officers and ensigns (midshipmen) - with intraocular lenses.

With visual acuity of one eye 0.09 (hereinafter according to Snellen's chart) and below or its blindness and with visual acuity of the other eye 0.3 and below, or when examining the eyesight of both eyes is 0.2 and below, citizens undergoing military service under contract are not recognized as fit for military service.
With visual acuity of one eye 0.09 and below or its blindness and with visual acuity of the other eye 0.4 and above, or visual acuity of one eye 0.3 with visual acuity of the other eye from 0.3 to 0.1 , there is an individual rating of the category (such personnel are recognized as either limited to military service or fit for military service with insignificant restrictions, taking into account their specialty and military post, ability to perform military service duties and health status).

If the visual acuity of one eye is 0.4 and from 0.3 to 0.1 in another eye, citizens undergoing contractual military service are considered to fit for military service with insignificant restrictions.

With persistent central scotomas on one or both eyes, the category of fitness for military service is determined depending on the status of the eye functions according to the relevant articles of the disease schedule.

\section{Conclusion}

Thus, taking into account the infectious theory of development of AMD with timely diagnostic and integrated approach to the treatment of AMD, the proposed therapy can be very effective, including the plan of maintaining the category of fitness for military service, which gives citizens the right to enter military service according to the visual functions. Further study of the role of each pathogenesis factor at all levels of the organism life cycle, from molecular to tissue, will allow developing fundamentally new and promising areas of AMD therapy, thereby preventing the development of various limitations in human life, promoting the preservation of active longevity in all areas of activity.

\section{References}

[1] E. G. Baranova, Y. V. Parkhomenko., O. N. Sizikova, PA. Ivanov and V. G. Krasnoperov, "Comparative analysis of the total lesion of the coronary bed, the severity of dyslipidemia and the fact of infection with herpes simplex virus 1,2 types, cytomegalovirus in patients with ischemic heart disease ", FarEastern Medical Journal, no. 4, 2006, pp. 5-7.

[2] M. M. Bikbov, R. R. Fajzrahmanov, A. L. Jarmukhametova. “Age-related macular degeneration”, Moscow: Aprel'; 2013.

[3] N. D. Yushchuk, Y. Y. Vengerov. "Infectious diseases: national guidance”, Moscow: GEOTAR-Media; 2009.

[4] V. A. Isakov, S. B. Rybalkin., M. G. Romantsov, "Herpesviral infection", Guidiance for doctors, St. Petersburg; 2006.

[5] S. A. Korotkikh, E. S. Knyazeva, L. V. Spiridonova, "Combined revascularization in dystrophic retinal diseases", Macula 2006: Thesis in collection of reports in the II All Russian seminar - "round table". Rostov-on-Don, 2006. pp. 337-339.

[6] E. S. Liebman, P. A. Tolmachev, E. V. Shakhova, "Epidemiological characteristics of disability due to the main forms of maculopathies", Macula 2006: Thesis in collection of reports in the II All Russian seminar - "round table". Rostovon-Don, 2006. pp. 15-17. 
[7] D. K. Lvov, "A guide to virology". Moscow: Medical Information Agency (MIA)"; 2013.

[8] L. K. Moshetova, A. P. Nesterov, E. A. Egorov, eds, "Agerelated macular degeneration. Clinical guidelines. Ophthalmology", Moscow: GEOTAR-Media; 2006.

[9] A. Rabson, A. Roit., P. Delves, "Fundamentals of Medical Immunology". Moscow: Mir Publishing House; 2006.

[10] G. Y. Stolyarenko, M. I. Tyurina, A. V. Khalaym, "Invasive therapy of the pathology of the macular area of the retina", Macula 2006: Thesis in collection of reports in the II All Russian seminar - "round table". Rostov-on-Don, 2006. pp. 379-380.

[11] V. K. Khavinson, S. V. Trofimova, "Peptide bioregulators in ophthalmology "; Saint Petersburg: Foliant, 2000.
[12] C. M. Cebulla, D. M. Miller D. A. Knight, B. R. Briggs, V. McGaughy, D. D. Sedmak, "Cytomegalovirus induces sialyl Lewis(x) and Lewis(x) on human endothelial cells", Transplantation.; vol. 69, N. 6, pp. 1202-1209. 2000.

[13] G. Hahn, R. Jores, E. S. Mocarski, Cytomegalovirus remains latent in a common precursor of dendritic and myeloid cells". Proceedings of the National Academy of Sciences of the United States of America. Vol. 95, N. 7, pp. 3937-3942, 1998.

[14] B. Slobedman, E. S. Mocarski, A. M. Arvin, E. D. Mellins, A. Abendroth, "Latent cytomegalovirus down-regulates major histocompatibility complex class II expression on myeloid progenitors”, Blood. Vol. 100, N.8, pp. 2867-2873, 2002. 